\title{
Application of Constant Amplitude Dynamic Tests for Life Prediction of Air Springs at Various Control Parameters
}

\author{
Tomaž Bešter* - Matija Fajdiga - Marko Nagode \\ University of Ljubljana, Faculty of Mechanical Engineering, Slovenia
}

Air spring manufactures use constant amplitude tests for the quality validation of air springs. The tests are very simple and the only information we get from them is that a spring is adequate if it passes the test and inadequate if it does not. One of the objectives of this article is to use these tests to make life predictions based on the standardised load spectrum. This prediction is made with force as the damage parameter. The second objective is to determine if it is possible to use experimental results obtained at one control parameter, e.g. force, to make life predictions for another control parameter, e.g. stress. With equations it is proved that such transformation is possible.

Keywords: vehicle suspension, air spring, load spectrum, dynamic tests, fatigue life

\section{O INTRODUCTION}

Air spring assembly consists of a piston, bellows, a bed plate and a bumper (Fig. 1). Pressure inside the bellows and the piston shape determines air spring characteristics. An air spring can have progressive spring characteristic, which is most suitable for transport vehicles that are loaded with various loads during exploitation [1].

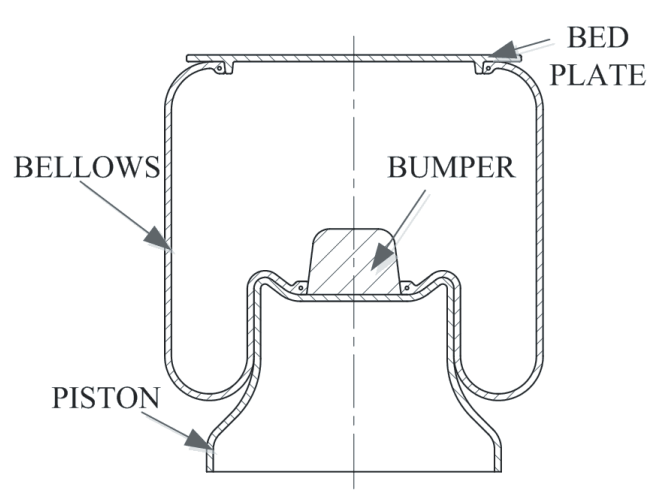

Fig. 1. Air spring

Air spring manufacturers have been testing springs with various static and dynamic experiments in order to verify spring quality. Dynamic tests usually have a constant amplitude and require a certain number of load cycles without critical damage on the air spring. If the spring successfully endures the test, critical damage on the spring does not occur and hence test results do not give exact information about fatigue life. In air spring fatigue tests, critical damage usually occurs on air spring bellows. In this article, the possibilities of obtaining SN-curves with modified constant amplitude tests will be examined. To make fatigue life predictions, loads on air springs and appropriate $\mathrm{SN}$-curves must also be determined.

\section{STANDARD LOAD SPECTRUM}

For transport vehicles, standard load spectra were determined [2] and [3] which define dynamic wheel force $F_{z, d y n}$ on transport vehicles. Standard load spectrum has $1.5 \times 10^{8}$ load cycles, which corresponds to $500000 \mathrm{~km}$ driving distance with 300 load cycles per kilometre. Standardised load spectra have three driving modes: straight driving, cornering and braking. Based on wheel force measurements during exploitation, standard load spectrum was determined with the level crossing method. Dynamic force $F_{z, d y n}$ in standardized load spectra is expressed with dynamic load factor $n_{\mathrm{z}}$ which represents ratio between dynamic and static load $n_{z}=F_{z, d y n} / F_{z, s t a}$. Dynamic load factor $n_{\mathrm{z}}$ depends on number of load cycles $N$ and driving mode (Table 1, Fig. 2). For arbitrary static load $F_{z, s t a}$ dynamic load $F_{z, d y n}$ can be determined with following equation:

$$
F_{z, d y n}=n_{z} \cdot F_{z, s t a} .
$$

Load ratio:

$$
R_{F}=\frac{F_{\min }}{F_{\max }}
$$

where $F_{\min }$ is minimal force, and $F_{\max }$ is maximal force. During exploitation this ratio is changing. Load ratio range for all driving modes has been presented in Table 2.

When deformations are small and materials have linear characteristic, load ratio is equal to stress ratio:

$$
R=R_{F}=R_{\sigma}=\frac{\sigma_{\min }}{\sigma_{\max }}
$$

where $\sigma_{\min }$ and $\sigma_{\max }$ are minimum and maximum stress. For high cycle fatigue, life calculation stress is 
usually used as the control parameter. When load ratio equals stress ratio, force can be used as the control parameter as well. On air springs, large deformations occur during exploitation and air spring bellows are made of polymer materials with nonlinear stress strain diagram [4] to [7]. Due to the geometric and material nonlinearities, Eq. (3) is not valid for air springs in general. This means fatigue life calculations with different control parameters will have different results. To compare fatigue life calculations with different parameters, SN-curves and Goodman diagrams would have to be determined for all control parameters in question. At least four experiments must be performed to design one Goodman diagram (Fig. 3). In this article, it will be shown how the Goodman diagram for one control parameter could be converted into the Goodman diagram for another control parameter that is not linearly dependent from the former parameter.

Table 1. Standard load spectra [2]

\begin{tabular}{|c|c|c|c|c|}
\hline \multirow[t]{2}{*}{ Driving mode } & \multirow{2}{*}{$\begin{array}{c}\begin{array}{c}\text { Dynamic } \\
\text { load factor }\end{array} \\
n_{z}\end{array}$} & \multicolumn{3}{|c|}{$\begin{array}{l}\text { Load spectrum parameters } \\
H_{g}=1.5 \times 10^{8}\end{array}$} \\
\hline & & $H$ & $H_{e}$ & SHAPE \\
\hline $\begin{array}{l}\text { Straight } \\
\text { driving }\end{array}$ & 2.0 & $0.96 \cdot H_{g}$ & $H \cdot 10^{-6}$ & $\begin{array}{c}\text { Linear } \\
\text { distribution }\end{array}$ \\
\hline $\begin{array}{l}\text { Cornering } \\
\text { Outer wheel } \\
\text { Inner wheel }\end{array}$ & $\begin{array}{l}1.5 \\
0.4\end{array}$ & $0.04 \cdot H_{g}$ & 50 & $\begin{array}{c}\text { Normal } \\
\text { distribution }\end{array}$ \\
\hline Breaking & 2.0 & $5 \cdot 10^{5}$ & $10^{4}$ & $\begin{array}{c}\text { Normal } \\
\text { distribution }\end{array}$ \\
\hline
\end{tabular}

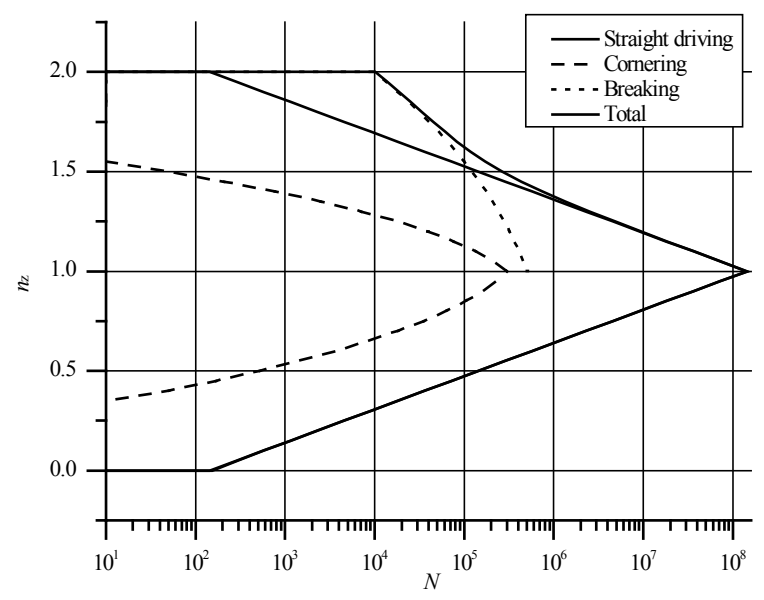

Fig. 2. Standard load spectra

Table 2. Load ratio ranges for various driving modes

\begin{tabular}{lc}
\hline Driving modes & $R_{F}$ \\
\hline Straight driving & 0 to 1 \\
\hline Cornering & 0.27 to 1 \\
\hline Breaking & 0.5 to 1 \\
\hline
\end{tabular}

\section{DYNAMIC EXPERIMENTS AND FATIGUE LIFE}

In the standardized load spectrum, load forces on wheels are defined. Wheel forces are transferred to air springs by suspension mechanism. As air spring forces are easily calculated, the easiest way to calculate fatigue life is to use force as a control parameter for the determination of SN-curves and the Goodman diagram. As load ratios of the standard load spectrum are in the range between 0 and 1, experiments for the determination of SN-curves and Goodman diagram must be in the same range.

For constant amplitude loads $F_{a 1 R 1}, F_{a 2 R 1}, F_{a 1 R 2}$ and $F_{a 2 R 2}$ the number of load cycles when critical damage occurs: $N_{1 R 1}, N_{2 R 1}, N_{1 R 2}$ and $N_{2 R 2}$ must be experimentally determined (Fig. 3). Those results allow to calculate $\mathrm{SN}$-curves slope:

$$
\begin{aligned}
& k_{R 1}=\frac{\log N_{a 1 R 1}-\log N_{a 2 R 1}}{\log F_{a 1 R 1}-\log F_{a 2 R 1}}, \\
& k_{R 2}=\frac{\log N_{a 1 R 2}-\log N_{a 2 R 2}}{\log F_{a 1 R 2}-\log F_{a 2 R 2}} .
\end{aligned}
$$

If fatigue limit is assumed to be at $N_{D}=2 \times 10^{6}$, amplitude fatigue strength can be calculated for both dynamic factors:

$$
\begin{aligned}
& F_{a D R 1}=F_{a 1 R 1}\left(\frac{N_{D}}{N_{1 R 1}}\right)^{\frac{1}{k_{R 1}}}, \\
& F_{a D R 2}=F_{a 1 R 2}\left(\frac{N_{D}}{N_{1 R 2}}\right)^{\frac{1}{k_{R 2}}} .
\end{aligned}
$$

From amplitude forces and load ratio, medium forces are calculated (for development of Eqs. (8) and (9) see the appendix):

$$
\begin{gathered}
F_{m D R 1}=\frac{F_{a D R 1}\left(1+R_{1}\right)}{\left(1-R_{1}\right)}, \\
F_{m D R 2}=\frac{F_{a D R 2}\left(1+R_{2}\right)}{\left(1-R_{2}\right)},
\end{gathered}
$$

and the gradient of the Goodman diagram:

$$
M=\frac{F_{a D R 2}-F_{a D R 1}}{F_{m D R 2}-F_{m D R 1}} .
$$

When the gradient of the Goodman diagram is known, equivalent amplitude load $F_{a 1 R 1}$ with load ratio $R_{1}$ can be calculated (Fig. 4 , for development of the Eq. (11) see the appendix): 

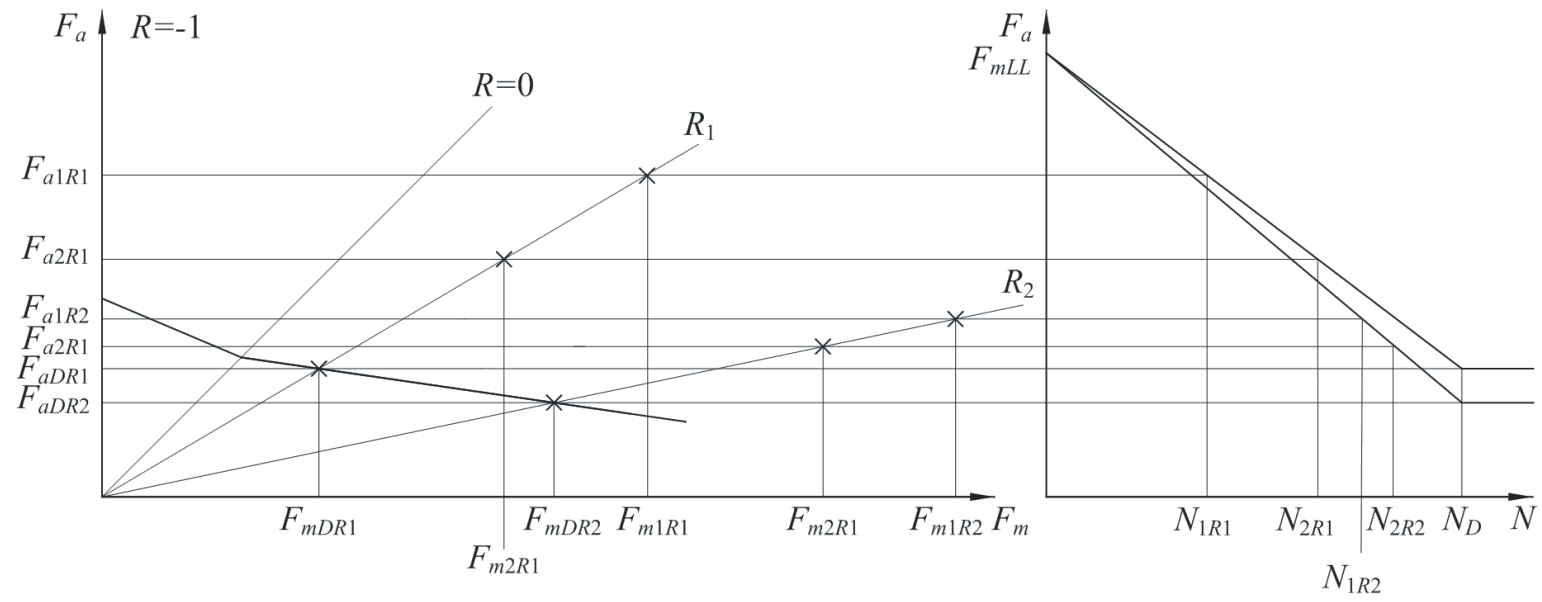

Fig. 3. Design of Goodman diagram

$$
F_{a 1 R 1}=F_{a 1 R i} \frac{\left(1+M \frac{\left(R_{i}+1\right)}{\left(R_{i}-1\right)}\right)}{\left(1+M \frac{\left(R_{1}+1\right)}{\left(R_{1}-1\right)}\right)} .
$$

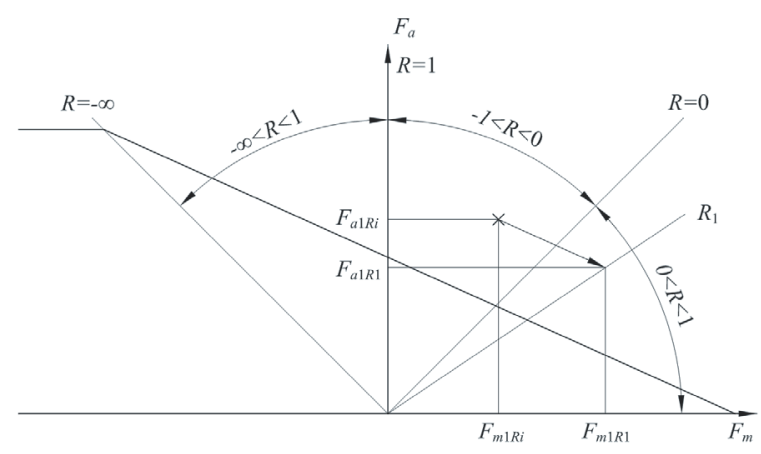

Fig. 4. Equivalent loads based on Goodman diagram [8]

Air spring manufacturers use various constant amplitude dynamic tests to validate quality and endurance of their air springs [9] and [10]. Two durability tests have been used in our research. In the first durability test, an air spring is loaded with the displacement amplitude $\pm 50 \mathrm{~mm}$, at the frequency $3.3 \mathrm{~Hz}$. The air spring must endure $2 \times 10^{6}$ load cycles without critical damage. In the second durability test, the displacement amplitude is $\pm 75 \mathrm{~mm}$ at the frequency $2 \mathrm{~Hz}$, with the air spring having to endure $10^{6}$ load cycles without critical damage. Both tests are stopped when the required number of load cycles is reached unless critical damage occurs first. If springs are tested until the critical damage occurs, two points on the SN-curve can be obtained. Air spring characteristic was measured (Fig. 5) to determine the appropriate force amplitude for any displacement amplitude.

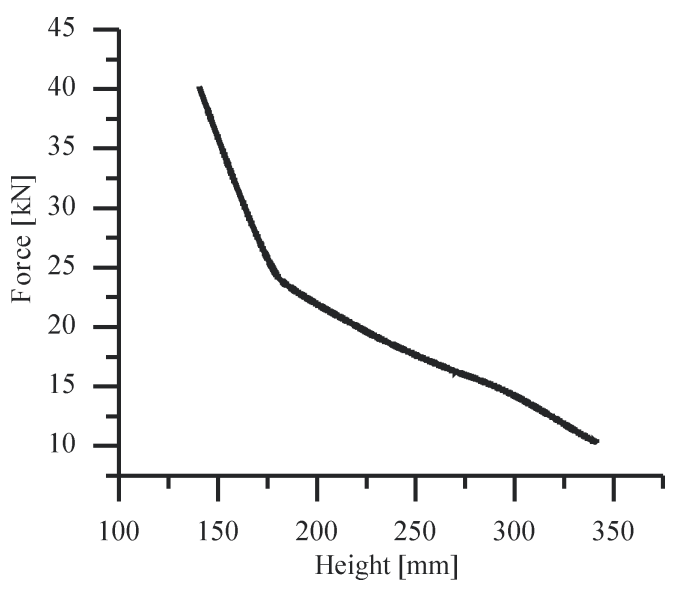

Fig. 5. Air spring characteristic

In the first durability test, the minimum required number of load cycles is the same as the fatigue limit. In the second durability test the minimum required number of load cycles is relatively near the fatigue limit. It is reasonable to use tests with higher amplitudes for determination of the remaining SNcurve points in order to reduce test time.

With two modified existing and two additional constant amplitude tests, two SN-curves and a Goodman diagram can be determined. This enables the transformation of any load to an equivalent load with the load ratio $R_{F}$ of a known SN-curve.

An SN-curve can be approximately determined with the tensile strength force $F_{M}$ and one dynamic test in the high cycle fatigue range because $\mathrm{SN}$-curves with various load factors intersect near the tensile strength 


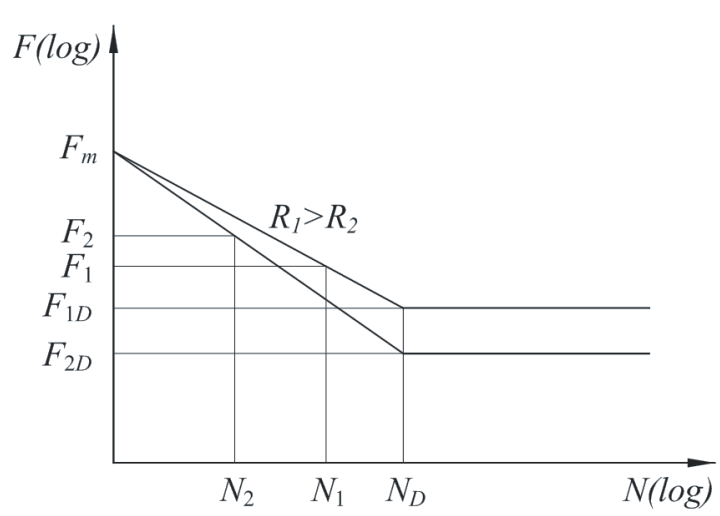

Fig. 6. Approximate $\mathrm{SN}$-curves [11]

force (Fig. 6). An air spring's maximum displacement is limited, therefore it is not possible to load a spring with a high enough load to cause the bellows to burst. The air spring bellows tensile strength cannot be directly determined, but it is possible to evaluate the tensile strength if pressure is increased in the spring's bellows until it bursts and the maximum force is used as the tensile strength [11].

Once SN-curves are determined, fatigue life can be calculated with one of the damage accumulation rules [8] and [11]. The most frequently used linear accumulation rules are the original and elementary Palmgren-Miner rule and the Haibach rule (Fig. 7). In all linear accumulation rules, damage $d_{i}$ is equal to the inverse number of load cycles when critical damage occurs. Total damage is:

$$
d=\sum_{i=1}^{n} \frac{1}{N_{i R}}
$$

In the described manner, damage was calculated for an air spring loaded with standardized load spectrum (Table 3). The results show significant differences between accumulation rules.

When material has stress in the linear region of the stress strain diagram and deformations are small, the stress ratio is equal to the load ratio. Air springs are made of material with nonlinear stress strain diagram and are submitted to large deformations in most load cycles of the standard load spectrum. To evaluate the significance of those nonlinearities, life predictions with stress as the damage parameter should be made.

Table 3. Fatigue life

\begin{tabular}{lccc}
\hline & $\begin{array}{c}\text { Basic } \\
\text { Palmgren- } \\
\text { Miner rule }\end{array}$ & $\begin{array}{c}\text { Elementary } \\
\text { Palmgren- } \\
\text { Miner rule }\end{array}$ & $\begin{array}{c}\text { Haibach } \\
\text { rule }\end{array}$ \\
\hline $\begin{array}{l}\text { Numumber of load } \\
\text { cycles }\end{array}$ & 9020073 & 3670064 & 5155576 \\
\hline $\begin{array}{l}\text { Relative number of } \\
\text { load cycles }\end{array}$ & 1 & 0.40688 & 0.57157 \\
\hline
\end{tabular}

\section{LIFE PREDICTION WHEN DYNAMIC EXPERIMETS HAVE DIFFERENT LOAD RATIOS}

\subsection{Determination of the Goodman Diagram from Four Dynamic Experiments with Different Load Ratios}

A pair of experiments with equal load ratios when force is used as the control parameter (Fig. 3) will not have equal stress ratios (Fig. 8) due to the nonlinear relation between force and stress. It is not possible to determine SN-curves and the Goodman diagram directly with four experiments that have four different stress ratios. It will be shown that it is possible to solve the problem with a system of equations. The solution of this system can give all the data needed to determine the SN-curves and the Goodman diagram

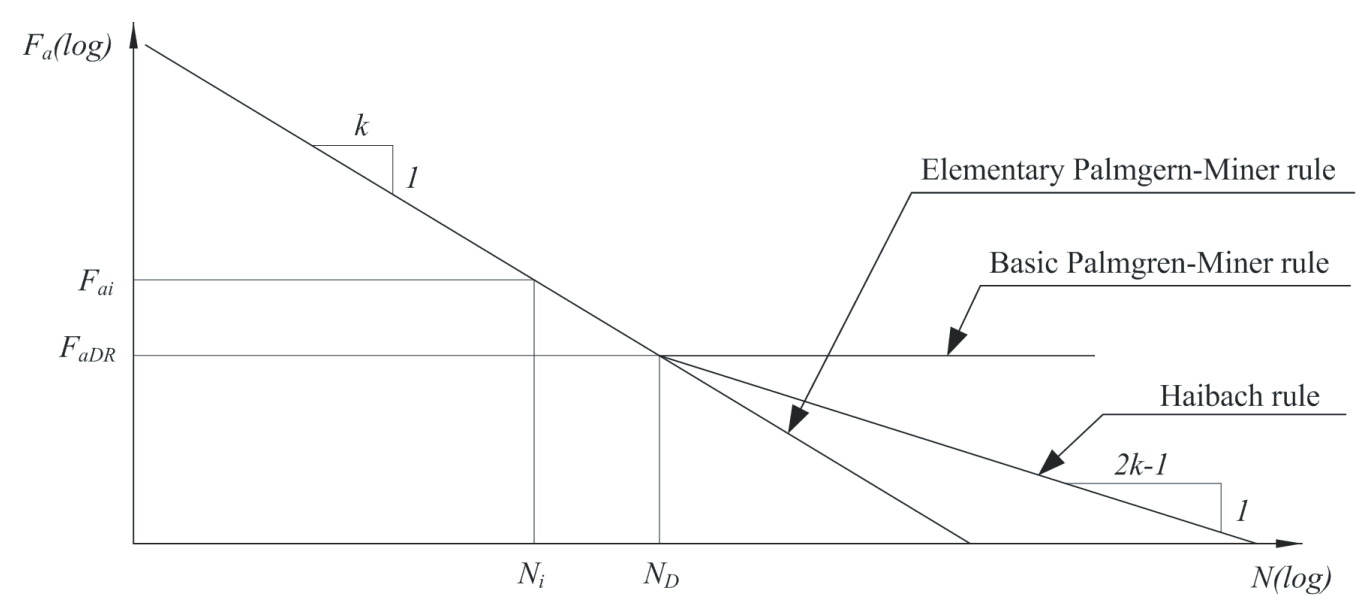

Fig. 7. SN-curves for original Palmgren-Miner, elementary Palmgren-Miner and Haibach rule [8] 


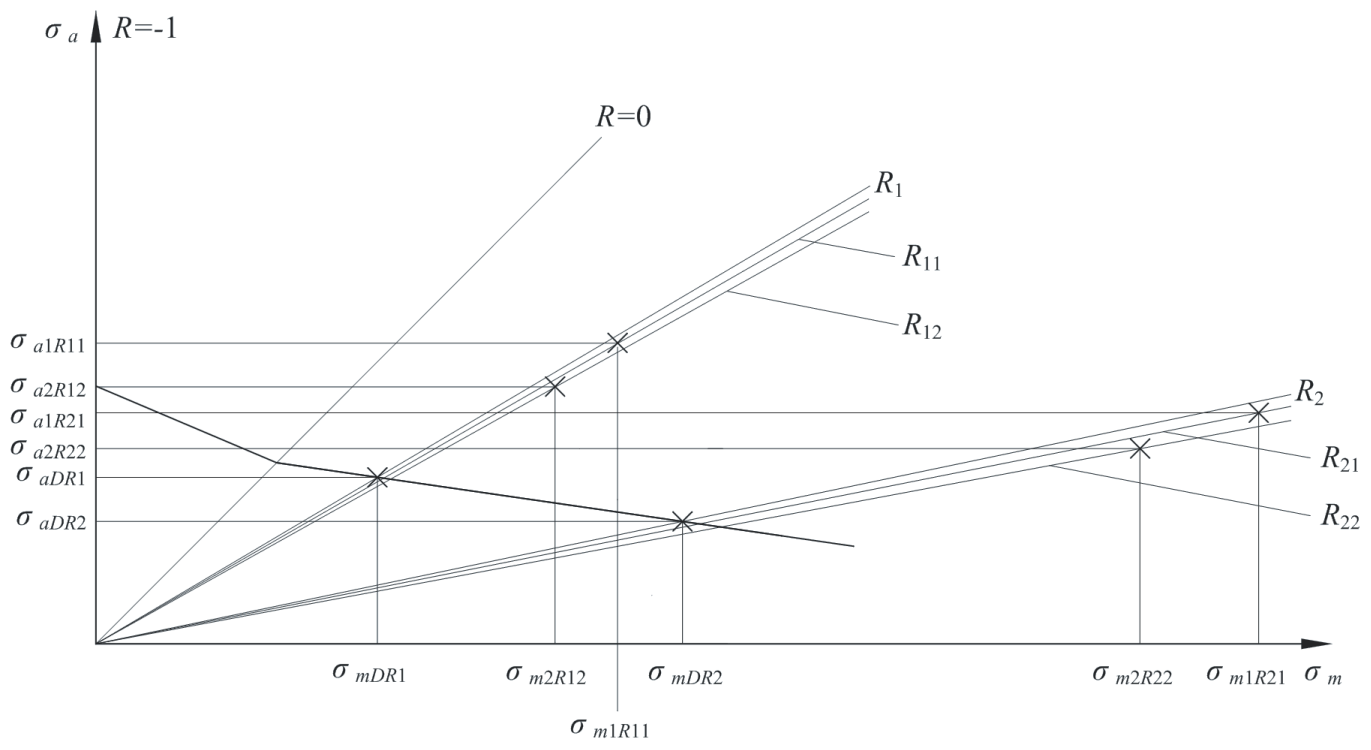

Fig. 8. Determination of the Goodman diagram from four dynamic tests

even if only four experiments with different stress ratios are available. Goodman diagram gradient $M$ depends on two fatigue limit medium and amplitude stresses $\sigma_{a D R 11}, \sigma_{a D R 21}, \sigma_{m D R 11}$ and $\sigma_{m D R 21}$ (Table 4). To determine those stresses, two $\mathrm{SN}$-curve gradients $k_{R 11}$ and $k_{R 21}$ are needed. To determine those gradients, it is necessary to obtain equivalent stresses with stress ratios $R_{11}$ and $R_{21}: \sigma_{m 2 R 11}, \sigma_{m 2 R 21}, \sigma_{a 2 R 11}$ and $\sigma_{a 2 R 21}$. To calculate equivalent stresses the gradient of the Goodman diagram $M$ is needed. There are eleven unknowns. Eleven equations can be set, hence the unknowns can be determined by the equation system solution:

$$
\begin{gathered}
\sigma_{m 2 R 11}=-\frac{\left(\sigma_{a 2 R 12}-M \sigma_{m 2 R 12}\right) \cdot\left(R_{11}+1\right)}{\left(\left(R_{11}-1\right)+M\left(R_{11}+1\right)\right)}, \\
\sigma_{m 2 R 21}=-\frac{\left(\sigma_{a 2 R 22}-M \sigma_{m 2 R 22}\right) \cdot\left(R_{21}+1\right)}{\left(\left(R_{21}-1\right)+M\left(R_{21}+1\right)\right)}, \\
\sigma_{a 2 R 11}=\frac{\sigma_{m 2 R 11}\left(1-R_{11}\right)}{\left(R_{11}+1\right)}, \\
\sigma_{a 2 R 21}=\frac{\sigma_{m 1 R 21}\left(1-R_{21}\right)}{\left(R_{21}+1\right)}, \\
k_{R 11}=\frac{\log N_{1 R 11}-\log N_{2 R 11}}{\log \sigma_{a 1 R 11}-\log \sigma_{a 2 R 11}} \\
k_{R 21}=\frac{\log N_{1 R 21}-\log N_{2 R 21}}{\log \sigma_{a 1 R 21}-\log \sigma_{a 2 R 21}}
\end{gathered}
$$

$$
\begin{gathered}
\sigma_{a D, R 11}=\sigma_{a 1 R 11}\left(\frac{N_{D}}{N_{1 R 11}}\right)^{\frac{1}{k_{R 11}}}, \\
\sigma_{a D R 21}=\sigma_{a 1 R 21}\left(\frac{N_{D}}{N_{1 R 21}}\right)^{\frac{1}{k_{R 21}}}, \\
\sigma_{m D R 11}=\frac{\sigma_{a D R 11}\left(1+R_{11}\right)}{\left(1-R_{11}\right)}, \\
\sigma_{m D R 21}=\frac{\sigma_{a D R 21}\left(1+R_{21}\right)}{\left(1-R_{21}\right)}, \\
M=\frac{\sigma_{a D R 21}-\sigma_{a D R 11}}{\sigma_{m D R 21}-\sigma_{m D R 11}} .
\end{gathered}
$$

It is difficult to determine stresses on air spring bellows because large deformations and material nonlinearity must be considered. Despite these difficulties, precise enough stress-strain analyses were made [12] to [14]. As stresses in air spring bellows can be determined, known quantities in the system of equations are medium and amplitude stresses (Table 4). With part of equations being nonlinear, the system of equations does not have a simple analytical solution, but it can be solved numerically. 
Table 4. Determination of the Goodman diagram from four dynamic tests- used quantities

\begin{tabular}{|c|c|c|c|}
\hline \multicolumn{2}{|r|}{ Known quantities } & \multicolumn{2}{|r|}{ Unknown quantities } \\
\hline$\sigma_{a 1 R 11}[\mathrm{MPa}]$ & Amplitude stress at $F_{a 1 R 1}[\mathrm{~N}]$ and $F_{m 1 R 1}[\mathrm{~N}]$ & $\sigma_{a D R 11}[\mathrm{MPa}]$ & Amplitude fatigue strength at $R_{11}$ \\
\hline$\sigma_{a 2 R 12}[\mathrm{MPa}]$ & Amplitude stress at $F_{\mathrm{a} 2 R 1}[\mathrm{~N}]$ and $F_{m 2 R 1}[\mathrm{~N}]$ & $\underline{\sigma_{a D R 21}[\mathrm{MPa}]}$ & Amplitude fatigue strength at $R_{R 21}$ \\
\hline$\sigma_{a 1 R 21}[\mathrm{MPa}]$ & Amplitude stress at $F_{a 2 R 1}[\mathrm{~N}]$ and $F_{m 2 R 1}[\mathrm{~N}]$ & $\sigma_{m D R 11}[\mathrm{MPa}]$ & Medium stress at $\sigma_{a D R 11}[\mathrm{MPa}]$ \\
\hline$\sigma_{\mathrm{a} 2 R 22}[\mathrm{MPa}]$ & Amplitude stress at $F_{\mathrm{a} 2 R 2}[\mathrm{~N}]$ and $F_{m 2 R 2}[\mathrm{~N}]$ & $\sigma_{m D R 21}[\mathrm{MPa}]$ & Medium stress at $\sigma_{a D R 21}[\mathrm{MPa}]$ \\
\hline$\sigma_{m 1 R 11}[\mathrm{MPa}]$ & Medium stress at $F_{m 1 R 1}[\mathrm{~N}]$ and $F_{a 1 R 1}[\mathrm{~N}]$ & $k_{R 11}$ & SN-curve gradient at $R_{11}$ \\
\hline$\sigma_{m 2 R 12}[\mathrm{MPa}]$ & Medium stress at $F_{m 2 R 1}[\mathrm{~N}]$ and $F_{\mathrm{a} 2 R 1}[\mathrm{~N}]$ & $k_{R 21}$ & SN-curve gradient at $R_{21}$ \\
\hline$\sigma_{m 1 R 21}[\mathrm{MPa}]$ & Medium stress at $F_{m 1 R 2}[\mathrm{~N}]$ and $F_{a 1 R 2}[\mathrm{~N}]$ & $\sigma_{\mathrm{a} 2 R 11}[\mathrm{MPa}]$ & Equivalent amplitude stress for $\sigma_{\text {a2R12 }}[\mathrm{MPa}]$ at $R_{11}$ \\
\hline$\sigma_{m 2 R 22}[\mathrm{MPa}]$ & Medium stress at $F_{m 2 R 2}[\mathrm{~N}]$ and $F_{\mathrm{a} 2 R 2}[\mathrm{~N}]$ & $\sigma_{\mathrm{a} 2 R 21}[\mathrm{MPa}]$ & Equivalent amplitude stress for $\sigma_{\text {a2R22 }}[\mathrm{MPa}]$ at $R_{21}$ \\
\hline$R_{11}$ & Stress ratio at $F_{a 1 R 1}[\mathrm{~N}]$ and $F_{m 1 R 1}[\mathrm{~N}]$ & $\sigma_{m 2 R 11}[\mathrm{MPa}]$ & Equivalent medium stress for $\sigma_{m 2 R 12}[\mathrm{MPa}]$ at $R_{11}$ \\
\hline$R_{12}$ & Stress ratio at $F_{\mathrm{a} 2 R 1}[\mathrm{~N}]$ and $F_{m 2 R 1}[\mathrm{~N}]$ & $\sigma_{m 2 R 21}[\mathrm{MPa}]$ & Equivalent medium stress for $\sigma_{m 2 R 22}[\mathrm{MPa}]$ at $R_{21}$ \\
\hline$R_{21}$ & Stress ratio at $F_{\mathrm{a} 2 R 1}[\mathrm{~N}]$ and $F_{m 2 R 1}[\mathrm{~N}]$ & $M$ & Goodman diagram gradient \\
\hline$R_{22}$ & Stress ratio at $F_{a 2 R 2}[\mathrm{~N}]$ and $F_{m 2 R 2}[\mathrm{~N}]$ & & \\
\hline$N_{1 R 11}$ & Number of load cycles when critical damage occurs at $F_{a 1 R 1}[\mathrm{~N}]$ & and $F_{m 1 R 1}[\mathrm{~N}]$ & \\
\hline$N_{2 R 12}$ & Number of load cycles when critical damage occurs at $F_{a 1 R 1}[\mathrm{~N}]$ & and $F_{m 1 R 1}[\mathrm{~N}]$ & \\
\hline$N_{1 R 21}$ & Number of load cycles when critical damage occurs at $F_{a 1 R 1}[\mathrm{~N}]$ & and $F_{m 1 R 1}[\mathrm{~N}]$ & \\
\hline$N_{2 R 22}$ & Number of load cycles when critical damage occurs at $F_{a 1 R 1}[\mathrm{~N}]$ & and $F_{m 1 R 1}[\mathrm{~N}]$ & \\
\hline$N_{D}$ & Fatigue limit & & \\
\hline
\end{tabular}

\subsection{Determination of the Goodman Diagram from Three Dynamic Experiments with Different Load Ratios}

Although it is possible to determine the Goodman diagram from four dynamic tests with different dynamic factors, it is easier to use just three dynamic tests, but we have to omit the calculation in the first step to determine $\sigma_{a D 1}, k_{R 1}$ and $M$. Equivalent stress $\sigma_{a 12}, \sigma_{m 12}$ and $\sigma_{a 13}, \sigma_{m 13}$ (Fig. 9, Table 5) can be calculated using following equations:

$$
\begin{aligned}
& \frac{\sigma_{a D 1}}{\sigma_{a 1}}=\left(\frac{N_{D}}{N_{1}}\right)^{\frac{1}{k_{1}}}, \\
& \frac{\sigma_{a D 1}}{\sigma_{a 12}}=\left(\frac{N_{D}}{N_{2}}\right)^{\frac{1}{k_{1}}}, \\
& \frac{\sigma_{a D 1}}{\sigma_{a 13}}=\left(\frac{N_{D}}{N_{3}}\right)^{\frac{1}{k_{1}}} .
\end{aligned}
$$

As $\sigma_{a 12}$ and $\sigma_{a 13}$ are not known in Eqs. (25) and (26), they must be expressed with known quantities. Based on the Goodman diagram equations can be written.

$$
\begin{aligned}
& \sigma_{a 12}-\sigma_{a 2}=M\left(\sigma_{m 12}-\sigma_{m 2}\right), \\
& \sigma_{a 13}-\sigma_{a 3}=M\left(\sigma_{m 13}-\sigma_{m 3}\right) .
\end{aligned}
$$

Medium stress can be written:

$$
\begin{gathered}
\sigma_{m 12}=\frac{\sigma_{a 12}\left(1+R_{1}\right)}{\left(1-R_{1}\right)}, \\
\sigma_{m 13}=\frac{\sigma_{a 13}\left(1+R_{1}\right)}{\left(1-R_{1}\right)} .
\end{gathered}
$$

If Eqs. (29) and (30) are inserted in Eqs. (27) and (28), equations for $\sigma_{a 12}$ and $\sigma_{a 13}$ can be written using $\sigma_{a 2}, \sigma_{a 3}, \sigma_{m 2}, \sigma_{m 3}, R_{1}$ and $M$, where $M$ is the only unknown:

$$
\begin{gathered}
\sigma_{a 12}=\frac{\left(\sigma_{a 2}-M \sigma_{m 2}\right)\left(1-R_{1}\right)}{\left(1-R_{1}\right)-M\left(1+R_{1}\right)}, \\
\sigma_{a 13}=\frac{\left(\sigma_{a 3}-M \sigma_{m 3}\right)\left(1-R_{1}\right)}{\left(1-R_{1}\right)-M\left(1+R_{1}\right)} .
\end{gathered}
$$

If logarithms from Eqs. (24), to (26) are calculated and Eqs. (31) and (32) are used for $\sigma_{a 12}$ and $\sigma_{a 13}$, a system of three equations and three unknowns is obtained:

$$
\begin{aligned}
& \log \sigma_{a D 1}-\log \sigma_{a 1}=\frac{1}{k_{1}}\left(\log N_{D}-\log N_{1}\right), \\
& \log \sigma_{a D 1}-\log \left(\frac{\left(\sigma_{a 2}-M \sigma_{m 2}\right)\left(1-R_{1}\right)}{\left(1-R_{1}\right)-M\left(1+R_{1}\right)}\right)= \\
& =\frac{1}{k_{1}}\left(\log N_{D}-\log N_{2}\right),
\end{aligned}
$$




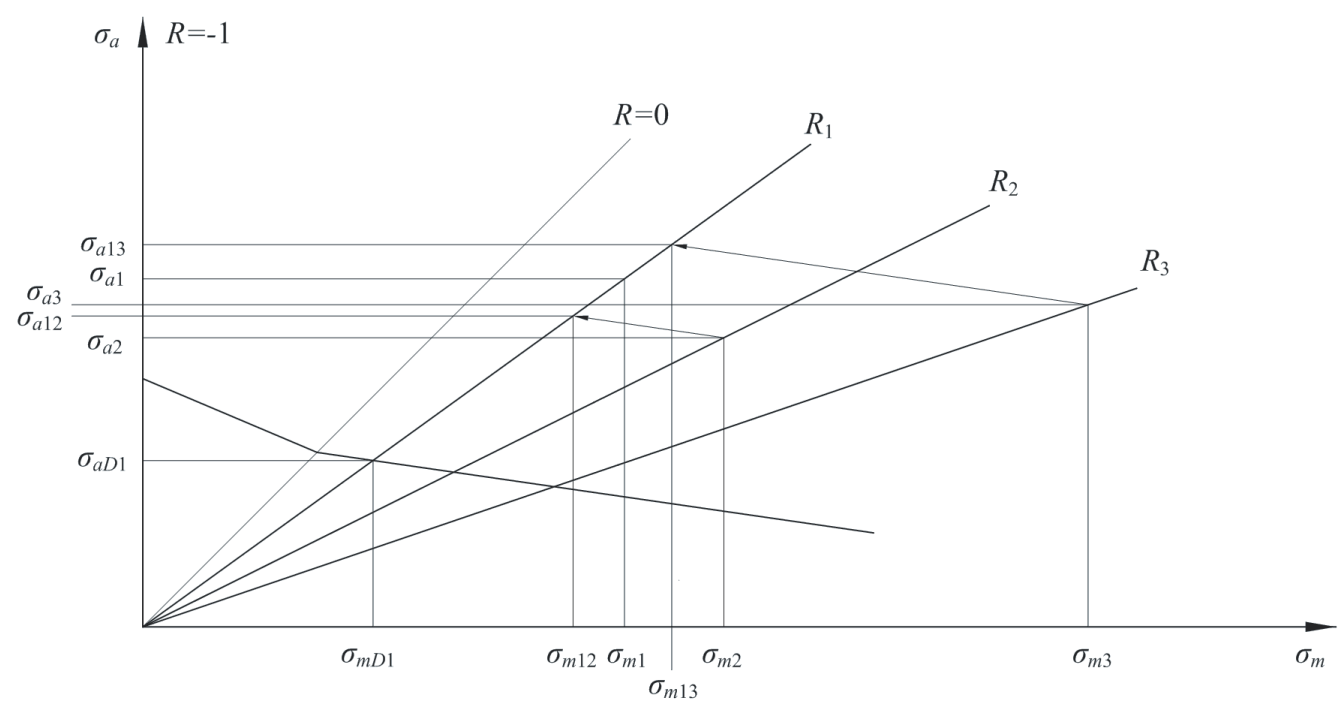

Fig. 9. Determination of the Goodman diagram from three dynamic tests

Table 5. Determination of the Goodman diagram from three dynamic tests - used quantities

\begin{tabular}{|c|c|c|c|}
\hline \multicolumn{2}{|r|}{ Known quantities } & \multicolumn{2}{|r|}{ Unknown quantities } \\
\hline$\sigma_{a 1}[\mathrm{MPa}]$ & Amplitude stress with stress ratio $R_{1}$ & $k_{1}$ & SN-curve gradient at $R_{1}$ \\
\hline$\sigma_{a 2}[\mathrm{MPa}]$ & Amplitude stress with stress ratio $R_{2}$ & $M$ & Goodman diagram gradient \\
\hline$\sigma_{\mathrm{a} 3}[\mathrm{MPa}]$ & Amplitude stress with stress ratio $R_{3}$ & $\sigma_{a D 1}[\mathrm{MPa}]$ & Amplitude fatigue strength at $R_{1}$ \\
\hline$\sigma_{m 1}[\mathrm{MPa}]$ & Medium stress with stress ratio $R_{1}$ & $\sigma_{m D 1}[\mathrm{MPa}]$ & Amplitude fatigue strength at $R_{1}$ \\
\hline$\sigma_{m 2}[\mathrm{MPa}]$ & Medium stress with stress ratio $R_{2}$ & $\sigma_{a 12}[\mathrm{MPa}]$ & Equivalent amplitude stress for $\sigma_{\mathrm{a} 2}$ at $R_{1}$ \\
\hline$\sigma_{m 3}[\mathrm{MPa}]$ & Medium stress with stress ratio $R_{3}$ & $\sigma_{\mathrm{a} 13}[\mathrm{MPa}]$ & Equivalent amplitude stress for $\sigma_{\mathrm{a} 3}$ at $R_{1}$ \\
\hline$R_{1}$ & Stress ratio at $\sigma_{a 1}$ and $\sigma_{m 1}$ & $\sigma_{m 12}[\mathrm{MPa}]$ & Equivalent medium stress for $\sigma_{m 2}$ at $R_{1}$ \\
\hline$R_{2}$ & Stress ratio at $\sigma_{\mathrm{a} 2}$ and $\sigma_{m 2}$ & $\sigma_{m 13}[\mathrm{MPa}]$ & Equivalent medium stress for $\sigma_{m 3}$ at $R_{1}$ \\
\hline$R_{3}$ & Stress ratio at $\sigma_{a 3}$ and $\sigma_{m 3}$ & & \\
\hline$N_{1}$ & Number of load cycles when critical damage occurs at $\sigma_{a 1}$ and $\sigma_{m 1}$ & & \\
\hline$N_{2}$ & Number of load cycles when critical damage occurs at $\sigma_{\mathrm{a} 2}$ and $\sigma_{m 2}$ & & \\
\hline$N_{3}$ & Number of load cycles when critical damage occurs at $\sigma_{a 3}$ and $\sigma_{m 3}$ & & \\
\hline$N_{D}$ & Fatigue limit & & \\
\hline
\end{tabular}

$$
\begin{aligned}
& \log \sigma_{a D 1}-\log \left(\frac{\left(\sigma_{a 3}-M \sigma_{m 3}\right)\left(1-R_{1}\right)}{\left(1-R_{1}\right)-M\left(1+R_{1}\right)}\right)= \\
& =\frac{1}{k_{1}}\left(\log N_{D}-\log N_{3}\right) .
\end{aligned}
$$

From Eq. (33) $\log \sigma_{a D 1}$ can be calculated:

$$
\log \sigma_{a D 1}=\log \sigma_{a 1}+\frac{1}{k_{1}}\left(\log N_{D}-\log N_{1}\right),
$$

and inserted into Eqs. (34) and (35). Thus two equations with two unknowns are obtained:

$$
\begin{aligned}
& \frac{1}{k_{1}}\left(\log \frac{N_{1}}{N_{2}}\right)+\log \left(\frac{\left(\sigma_{a 2}-M \sigma_{m 2}\right)\left(1-R_{1}\right)}{\left(1-R_{1}\right)-M\left(1+R_{1}\right)}\right)=\log \sigma_{a 1} \\
& \frac{1}{k_{1}}\left(\log \frac{N_{1}}{N_{3}}\right)+\log \left(\frac{\left(\sigma_{a 3}-M \sigma_{m 3}\right)\left(1-R_{1}\right)}{\left(1-R_{1}\right)-M\left(1+R_{1}\right)}\right)=\log \sigma_{a 1}
\end{aligned}
$$

There is no simple analytical solution for Eqs. (37) and (38) but it is possible to calculate $k_{1}$ and $M$ numerically. When $k_{1}$ is calculated, fatigue limit $\sigma_{a D 1}$ can be calculated with Eq. (36). With the Goodman diagram gradient $M$, any stress $\sigma_{a 1 R i}$ with arbitrary stress ratio $R_{i}$ can be transformed to equivalent stress $\sigma_{a 1 R 1}$ with stress ratio $R_{1}$ and known SN-curve gradient $k_{1}$ : 


$$
\sigma_{a 1 R 1}=\sigma_{a 1 R i} \frac{\left(1+M \frac{\left(R_{i}+1\right)}{\left(R_{i}-1\right)}\right)}{\left(1+M \frac{\left(R_{1}+1\right)}{\left(R_{1}-1\right)}\right)} .
$$

This enables to calculate equivalent stress for any load, e.g. $\sigma_{a 12}, \sigma_{a 13}$, etc. (Fig. 9).

\section{CONCLUSION}

Unchanged durability tests cannot be used to make fatigue life predictions, because unchanged tests are stopped when the required number of load cycles is reached. If those tests are performed until critical damage is reached and additional constant amplitude tests are made, two SN-curves, Goodman diagram and fatigue life prediction can be made. Fatigue life prediction was made with force as damage parameter using three damage accumulation rules. Various damage accumulation methods gave significantly different results. The elementary Palmgren-Miner rule and the Haibach modification take into account even loads that are smaller than the fatigue limit therefore those rules give shorter fatigue life predictions than the basic Palmgren-Miner rule. No tests were made with loads smaller than the fatigue limit therefore no estimation about the accuracy of damage accumulation rules in this range can be made.

Due to geometric and material nonlinearities, fatigue life calculations with force as the control parameter may be significantly different than fatigue life calculations with stress as the control parameter. The comparison of life predictions using different control parameters would be very time consuming and expensive as we would have to experimentally determine Goodman diagrams for all control parameters in question. With the equations we proved it is possible to transform the Goodman diagram for one control parameter, e.g. force, into the Goodman diagram for another control parameter, e.g. stress. The transformation of the Goodman diagram to arbitrary control parameters makes further research of the influence of control parameters on fatigue life much quicker and less expensive.

\section{ACKNOWLEDGMENT}

The authors appreciate the support provided by the Veyance Technologies Europe, d.o.o.

\section{APPENDIX}

Development of the medium force equation (Eqs. (8) and (9). Analogue equations are used for medium stress (Eqs. (29) and (30)).

$F_{D \text { max }}$ - maximum force

$F_{D \text { min }}-$ minimum force

$F_{m D R i}$ - medium force with load ratio $R_{i}$ $F_{a D R i}$ - amplitude force with load ratio $R_{i}$

$$
\begin{gathered}
R_{i}=\frac{F_{D \min }}{F_{D \max }}=\frac{F_{m D R i}-F_{a D R i}}{F_{m D R i}+F_{a D R i}}, \\
R_{i} \cdot F_{m D R i}+R_{i} \cdot F_{a D R i}=F_{m D R i}-F_{a D R i}, \\
R_{i} \cdot F_{m D R i}-F_{m D R i}=-R_{i} \cdot F_{a D R i}-F_{a D R i}, \\
F_{m D R i}\left(R_{i}-1\right)=-F_{a D R i}\left(R_{i}+1\right), \\
F_{m D R i}=-F_{a D R i} \frac{\left(R_{i}+1\right)}{\left(R_{i}-1\right)}=F_{a D R i} \frac{\left(1+R_{i}\right)}{\left(1-R_{i}\right)} .
\end{gathered}
$$

Development of the equivalent amplitude force equation (Eq (11), Fig. 4). Analogue equation is used for equivalent amplitude stress (Eq. (39)).

$F_{a 1 R i}$ - amplitude load with load ratio $R_{i}$

$F_{a 1 R 1}$ - equivalent amplitude load with load ratio $R_{1}$

$F_{m 1 R i}$ - medium load with load ratio $R_{i}$

$F_{m 1 R 1}$ - equivalent medium load with load ratio $R_{1}$

$$
\begin{gathered}
M=\frac{F_{a 1 R 1}-F_{a 1 R i}}{F_{m 1 R 1}-F_{m 1 R i}}, \\
F_{a 1 R 1}-F_{a 1 R i}=M\left(F_{m 1 R 1}-F_{m 1 R i}\right), \\
F_{a 1 R 1}-F_{a 1 R i}=M\left(-F_{a 1 R 1} \frac{\left(R_{1}+1\right)}{\left(R_{1}-1\right)}-F_{m 1 R i}\right), \\
F_{a 1 R 1}+M \cdot F_{a 1 R 1} \frac{\left(R_{1}+1\right)}{\left(R_{1}-1\right)}=F_{a 1 R i}-M \cdot F_{m 1 R i}, \\
F_{a 1 R 1}\left(1+M \frac{\left(R_{1}+1\right)}{\left(R_{1}-1\right)}\right)=F_{a 1 R i}+M \cdot F_{a 1 R i} \frac{\left(R_{i}+1\right)}{\left(R_{i}-1\right)}, \\
F_{a 1 R 1}=F_{a 1 R i} \frac{\left(1+M \frac{\left(R_{i}+1\right)}{\left(R_{i}-1\right)}\right)}{\left(1+M \frac{\left(R_{1}+1\right)}{\left(R_{1}-1\right)}\right)}
\end{gathered}
$$




\section{REFERENCES}

[1] Pirnat, M., Savšek, Z., Boltežar, M. (2011). Measuring dynamic loads on a foldable city bicycle. Strojniški vestnik - Journal of Mechanical Engineering, vol. 57, no. 1, p. 21-26, DOI:10.5545/sv-jme.2009.149.21-26, 2011.

[2] Neugebauer, J.R., Grubisic, V., Fischer, G. (1989). Procedure For Design Optimization And Durability Life Approval Of Truck Axles And Axle Assemblies. SAE Technical Paper, 892535.

[3] Heuler, P., Klätschke, H. (2005). Generation and use of standardised load spectra and load-time histories. International Journal of Fatigue, vol. 27, no. 8, p. 974990, DOI:10.1016/j.ijfatigue.2004.09.012.

[4] Oman, S., Nagode, M., Fajdiga, M. (2009).The material characterization of the air spring bellow sealing layer. Materials \& Design, vol. 30, no. 4, p. 1141-1150, DOI:10.1016/j.matdes.2008.06.035.

[5] Shaw, M.T., MacKnight, W.J. (2005). Introduction to Polymer Viscoelasticity, 3rd ed. John Wiley \& Sons, Hoboken, DOI:10.1002/0471741833.

[6] Tschoegl, N.W., Knauss, W.G., Emri, I. (2002). The effect of temperature and pressure on the mechanical properties of thermo- and/or piezorheologically simple polymeric materials in thermodynamic equilibrium - A critical review. Mechanics of Time-Dependent Materials, vol. 6, no. 1, p. 53-99, DOI:10.1023/A:1014421519100.

[7] Gent, A. (2012). Engineering With Rubber. Carl Hanser Verlag, Munich, DOI:10.3139/9783446428713.

[8] LMS Falancs Theory Manual version 2.9. (2000). LMS Durability Technologies, Leuven.

[9] Air Spring - Instaltion Requirement (2008). Mack Trucks, Allentown.

[10] Durability-Flex Life (2009). Goodyear Tire and Rubber Company, Green.

[11] Ellyin, F. (1997). Fatigue Damage, Crack Growth And Life Predicition. Chapman\&Hall, London.

[12] Oman, S., Fajdiga, M., Nagode, M. (2010). Estimation of air-spring life based on accelerated experiments. Materials and Design, vol. 31, no. 8, p. 3859-3868, DOI:10.1016/j.matdes.2010.03.044.

[13] Hackenschmidt, R., Alber-Laukant, B., Rieg, F. (2011). Simulating nonlinear materials under centrifugal forces. Strojniški vestnik - Journal of Mechanical Engineering, vol. 57, no. 7-8, p. 531-538, DOI:10.5545/ sv-jme.2011.013.

[14] Oman, S., Nagode, M. (2013). On the influence of the cord angle on air-spring fatigue life. Engineering Failure Analysis, vol. 27, p. 61-73, DOI:10.1016/j. engfailanal.2012.09.002. 